\title{
Hollow waveguide as an online microliter spectroscopy sensor for gas chromatography
}

\author{
Andrei Deev, Sheng Wu, Yongchun Tang \\ PEER Center, Caltech, 738 Arrow Grand Circle, Covina, CA 91722
}

\begin{abstract}
Quantum Cascade laser is coupled into Hollow Waveguide as an inline sensor after GC separation. We show the sensor has high ppbV sensitivity for carbon and other species, yet maintaining the GC peaks. OCIS Code: $300.6260,300.1030$
\end{abstract}

\section{Introduction}

Infrared (IR) sensors using gold plated tube and traditional broadband IR source have been coupled to Gas Chromatography (GC-IR) to provide further identification of chemicals after GC separation column. There have been great improvements in the performances of Quantum Cascade lasers in the mid-IR and far-IR in the past several years, and these advances in IR light sources promise new performances for such GC-IR sensors. We give a scheme integrating a Hollow Waveguide (HW) with long optical absorption path and yet microliter sample volume, and a Quantum Cascade (QC) IR laser source to give unprecedented performances to GC-IR sensors. We present the preliminary results of using such sensor for ${ }^{13} \mathrm{C}$ isotope detection for hydrocarbon compounds.

\section{Experiment setup}

The system schematic is given in figure 1, the Hollow Waveguide for IR transmission (Polymicro Technology, HWEA300750, typically 0.5 meter long and 300 micron ID) is coupled to the GC line with a special custom designed coupling device that has channels with cross section that matches the capillary and minimizes dead volume, and the HW and GC capillary are coupled to the coupling device with GC ferrules connectors. The coupling device also has a $\mathrm{CaF} 2$ window that allows coupling of Infra-red light into the $\mathrm{HW}$ at almost orthogonal direction to the GC line. Similar coupling device is used for the exit of the HW, where the existing IR light is coming out the other CaF2 window and detected by an IR detector whereas the gas is coupled out of the coupling devices for further online detection. The total volume of the HW is only $35 \mu \mathrm{l}$ and yet this HW has an optical path length of 0.5 meter. In commercial GC-IR setup, the gold plated waveguide for IR transmission usually has an ID of $1 \mathrm{~mm}$, and the length is $10 \mathrm{~cm}$ to $15 \mathrm{~cm}$ long or a total volume about $100 \mu \mathrm{l}$, and due to the larger ID they need makeup gas and the sensor destroys the peak shape.

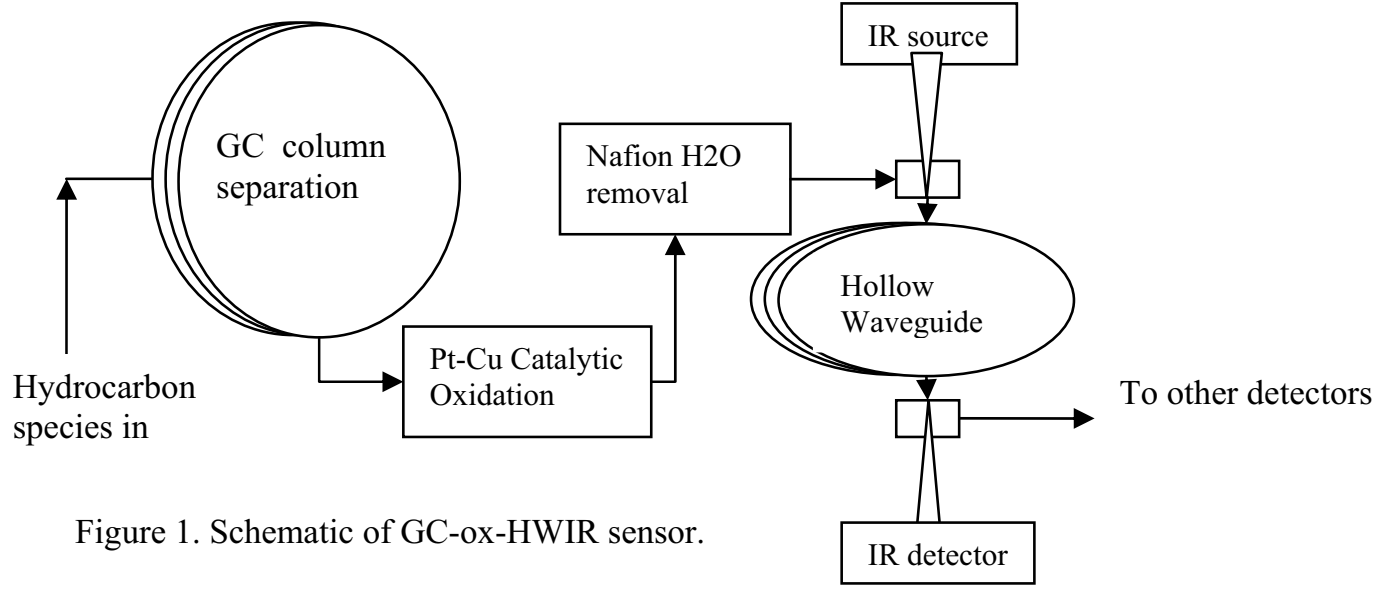

Compared to previous results where gas sample and IR injections happen at the same input and exit ports for hollow waveguides ${ }^{1-3}$, our design emphasizes the ability to couple with GC line, and maintain the gas peaks before and after the IR gas sensing measurement. The ability to couple to GC columns enhances the HW IR spectroscopy sensor capability because the GC column purifies the samples by separating each sample from a mixture. The purified samples out of GC column give much cleaner background in IR spectra and make distinguishing trace amount of isotopomer practical. The ability to maintain the gas peak 
shape after the IR sensor gives the possibilities to conduct other precise comparison measurement with the same gas samples without introducing errors due to gas splitting.

To detect $\mathrm{CO}_{2}$ isotpomers, i.e. ${ }^{13} \mathrm{CO}_{2}$, we used a Quantum Cascade (QC) laser at 4.45 micron (Alps Lasers, Switzerland). It is coupled into the $\mathrm{HW}$ with an uncoated $\mathrm{F} \#=1$ double convex $\mathrm{ZnSe}$ lens, and light is coupled into the HW with a focusing condition that matches the Numeric Aperture $(\mathrm{NA}=0.04)$ of the HW. We achieved a coupling efficiency of over $40 \%$ and the signal out of the HW is detected with a fast IR detector. We have tried IR LED and IR lamp, but the coupling efficiency is very low, e.g. well under $5 \%$. We therefore conclude that QC laser gives the good results because of its small etendue that allow it efficiently couple into HW. The QC laser is a DFB laser operating at $200 \mathrm{KHz}$ pulse repetition rate with $20 \mathrm{nsec}$ pulse width and peak power is about $1 \mathrm{~mW}$. We ramp the DFB laser bias current to scan the wavelength of the DFB laser at $500 \mathrm{~Hz}$. We integrate the absorption feature of ${ }^{13} \mathrm{CO}_{2}$ molecule in each ramp and then average 100 ramps to get an absorption value with an absorption baseline noise of $10^{-3}$. We could collect absorption data at $5 \mathrm{~Hz}$, and this is enough for use with GC peak area measurement.

\section{Results}

Figure 2 gives the GC spectra detected with HWIR for ${ }^{13} \mathrm{CO}_{2}$ and TCD detectors for total $\mathrm{CO} 2\left({ }^{13} \mathrm{CO}_{2}\right.$ $\left.+{ }^{12} \mathrm{CO}_{2}\right)$, and the three peaks corresponds to the methane, ethane and propane gases from a refinery gas mixture. The HWIR detector is tuned to detect ${ }^{13} \mathrm{CO}_{2}$ and the peaks correspond to the ${ }^{13} \mathrm{C}$ concentrations of methane, ethane and propane. We have achieved signal to noise ratio well over 1,000:1 for the HWIR sensor for ${ }^{13} \mathrm{CO}_{2}$, and therefore making this instrument ideal for ${ }^{13} \mathrm{C}$ isotopomers detection for $\mathrm{C} 1, \mathrm{C} 2$ and $\mathrm{C} 3$ gases in the field. The same gas line after the HWIR sensor is routed to a TCD detector without makeup gas and the TCD signal is also recorded for the 3 peaks, with their time position only delayed about 8 seconds.

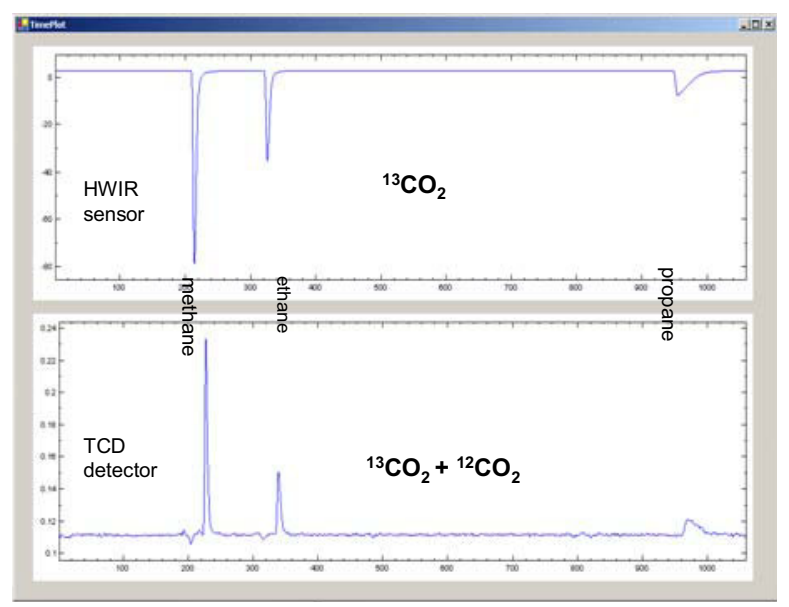

Figure 2. GC effluent peaks for Methane (C1), Ethane (C2) and Propane (C3) mixture recorded by HWIR sensor for $13 \mathrm{CO} 2$ (top one) and then by TCD detector for total $13 \mathrm{CO} 2+12 \mathrm{CO} 2$ (bottom one). The peaks from TCD dector still maintain their shape and sharpness after the online HWIR sensor. The GC peaks are recorded under isothermal conditions at $35^{\circ} \mathrm{C}$ and the flow rate is set at $1 \mathrm{ml} / \mathrm{min}$.

\section{Reference:}

1. G. J. P. Fetzer, A.S., " Mid-infrared laser absorption spectroscopy in coiled hollow optical waveguides," Lasers and Electro-Optics Society, 2003. LEOS 2003. The 16th Annual Meeting of the IEEE 1, 464-465 (2003).

2. C. d. M. Charlton, F. Inberg, A. Croitoru, N. Mizaikoff, B. , " Hollow-waveguide gas sensing with room-temperature quantum cascade lasers," IEE Proceedings, Optoelectronics 150, 306-309 (2003).

$3 . \quad$ R. H. M. R. L. Kozodoy, and J. A. Harrington, "Small-Bore Hollow Waveguide Infrared Absorption Cells for Gas Sensing," Appl. Spectrosc. 50, 415-417 (1996). 